\title{
Langzeitkonten - Neue Chancen für die Gestaltung von Arbeitszeiten und Lebensläufen?
}

Langzeitkonten stellen eine „Verlängerung“ des seit Jahrzehnten etablierten Gleitzeitkontos dar. Gleitzeitkonten sind ein Ansatzpunkt, um die alltägliche Vereinbarkeit von Arbeitszeiten und lebensweltlichen Anforderungen zu erleichtern. Langzeitkonten könnten die weitergehende Möglichkeit eröffnen, die mit den Gleitzeitkonten praktizierte „kleine“ Form der „Balance von Arbeit und Leben“ über längere Zeiträume auszudehnen - gegebenenfalls sogar über das gesamte Erwerbsleben hinweg. Damit stellt sich die Frage, ob und unter welchen Bedingungen sich dieses Potenzial in der betrieblichen Praxis entfalten kann.

\section{Potenziale von Langzeit- konten - neue Optionen der Lebensgestaltung}

Langzeitkonten werden in einer Vielzahl von sozialwissenschaftlichen Veröffentlichungen, Managementmagazinen und Zeitungsartikeln als wichtiges Instrument flexibler Arbeitszeitpolitik bezeichnet (IW 2006; DIHK 2004). Dieser neue Typ von Arbeitszeitkonten umfasst die langfristige Ansammlung von abweichenden Arbeitszeiten und teilweise von Entgeltbestandteilen auf individuellen Konten, die zu einem späteren Zeitpunkt für vereinbarte Zwecke wieder entnommen werden können (vgl. Seifert 2005). Unter Langzeitkonten verstehen wir im Folgenden Arbeitszeitkonten mit einem Ausgleichszeitraum, der länger als ein Jahr ist. Die Grundidee ist einfach: Mitarbeiter können über viele Jahre hinweg Überstunden oder Entgeltanteile auf einem Zeitwertkonto „sparen“ und zu einem späteren Zeitpunkt für bezahlte Freistellungen nutzen, etwa für Kinderbetreuung, Weiterbildung, Sabbaticals oder den vorzeitigen Ausstieg aus dem Erwerbsleben. Für die Betriebe werden auf diese Weise größere Flexibilitätsspielräume möglich. In Zeiten hoher Nachfrage können sie über längere Zeit auf die Mehrarbeit ihrer Mitarbeiter zurückgreifen, ohne kurzfristigen Zeitausgleich oder Zuschläge und Prämien gewähren zu müssen.

Auch den Beschäftigten können Langzeitkonten neue Chancen bieten. Im Unterschied zu anderen Formen der Arbeitszeitflexibilisierung wie etwa Teilzeitarbeit bleibt das individuelle Einkommen bei Ar- beitszeitreduzierungen (weitgehend) unberührt. Gerade in der Familienphase, wenn Kinder den Zeit- und Geldbedarf des Haushalts gleichermaßen ansteigen lassen, bieten Zeitentnahmen aus dem Langzeitkonto Vorteile. Sie eröffnen die Option, phasenweise weniger $\mathrm{zu}$ arbeiten, ohne auf Einkommen verzichten zu müssen. Ähnliches gilt für andere Ziele, wie Weiterbildung und Studium, die Pflege von Familienangehörigen, ehrenamtliches Engagement, vorzeitigen bzw. gleitenden Ruhestand oder Auszeiten für Erholung, Entspannung oder persönliche Interessen. Solche Arbeitszeitoptionen können es den Beschäftigten ermöglichen, berufliche und außerberufliche Anforderungen besser über den Lebenslauf zu verteilen, kumulierende Belastungen zu vermeiden und Zeiträume für lebenslanges Lernen, Erholung, Freizeit und soziales Engagement zu schaffen. In diesem Sinne bergen sie ein großes Potenzial für die Lebensqualität der Beschäftigten, den Erhalt ihrer Arbeitsmarktund Beschäftigungsfähigkeit und die bessere Vereinbarkeit von beruflichen und außerberuflichen Anforderungen (Anxo et al. 2008). Vor dem Hintergrund zunehmender Unsicherheiten und Risiken in den Erwerbsverläufen könnten sie zugleich einen Beitrag zur Vermeidung wie Überbrückung von Phasen beruflicher oder privater Krisen leisten.

In der gesellschaftspolitischen Debatte wecken Lebensarbeitszeitansätze derzeit große Aufmerksamkeit. ${ }^{1}$ Ihnen wird oft eine wichtige Rolle bei der Bewältigung der demografisch bedingten Veränderungen in der Alters- und Beschäftigungsstruktur der Bevölkerung zugedacht (Brandl et al. 2008). Stichworte sind hier die Erhöhung der Arbeitsmarkt- und Beschäftigungs- fähigkeit sowie bessere Möglichkeiten zur Vereinbarkeit von Beruf und Familie. Der Siebte Familienbericht des Familienministeriums fordert in diesem Zusammenhang explizit eine Neuorganisation der Zeitverteilung im Erwerbsverlauf in Form flexibler „Optionszeiten“ für Pflege und Betreuung („Care-Zeiten“), berufliche Weiterbildung („Bildungszeiten“) oder zivilgesellschaftliches Engagement („Sozialzeiten“) (BMFSFJ 2006).

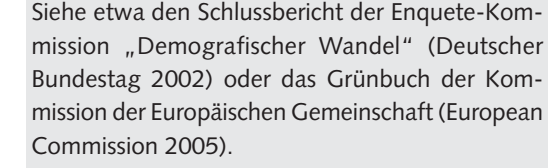
mission "Demografischer Wandel" (Deutscher Bundestag 2002) oder das Grünbuch der Kommission der Europäischen Gemeinschaft (European Commission 2005).

Philip Wotschack, Dipl. soz., Mitarbeiter der Abteilung Ausbildung und Arbeitsmarkt am Wissenschaftszentrum Berlin für Sozialforschung (WZB). Arbeitsschwerpunkte: Arbeitsorganisation und Arbeitszeit, Betriebliche Lebenslaufpolitik, Zeitorganisation von Paaren.

e-mail:wotschack@wzb.eu Eckart Hildebrandt, Prof. Dr., bis Mai 2008 Mitarbeiter der Abteilung Ausbildung und Arbeitsmarktpolitik am WZB. Arbeitsschwerpunkte: Zukünfte der Arbeit und Nachhaltigkeit, flexible Arbeitszeiten, alltägliche und biografische Lebensführung, Transformation der industriellen Beziehungen. e-mail: seeloewe@wzb.eu

Franziska Scheier, Dipl.-Sowi., Mitarbeiterin der Abteilung Ausbildung und Arbeitsmarkt am WZB. Arbeitsschwerpunkte: Arbeit und Organisation, betriebliche Lebenslauf- und Arbeitszeitpolitik, Arbeitnehmervertretungen und Gewerkschaften. e-mail: scheier@wzb.eu 
Inwieweit und unter welchen Bedingungen dieses Potenzial von Langzeitkonten in der betrieblichen Praxis eingelöst wird und tatsächlich neue Chancen der Lebensgestaltung für die Beschäftigten eröffnet, ist die leitende Frage unserer Untersuchung. Um sie zu beantworten, haben wir am Wissenschaftszentrum Berlin für Sozialforschung (WZB) von 2006 bis 2008 die Verbreitung und Nutzung von Langzeitkonten in deutschen Unternehmen untersucht. ${ }^{2}$ Wir unterscheiden dabei auf der einen Seite zwischen „Lebensarbeitszeitkonten", bei denen das Zeitguthaben für den vorzeitigen Ausstieg aus dem Erwerbsleben genutzt werden kann. Auf der anderen Seite stehen „optionale Langzeitkonten“, die Entnahmen während des Erwerbslebens vorsehen: für Weiterbildung, Familie oder andere Zwecke. Die Funktion von „Lebensarbeitszeitkonten" besteht darin, den Beschäftigten (und dabei vor allem den Älteren) flexible Übergänge in den Ruhestand zu ermöglichen. Diese Option gewinnt für Betrieb und Beschäftigte vor allem im $\mathrm{Zu}$ ge des Auslaufens der staatlich geförderten Altersteilzeit sowie der Anhebung des gesetzlichen Renteneintrittsalters an Bedeutung. Unter dem Gesichtspunkt präventiv angelegter Arbeitszeitoptionen, die - ganz im Sinne der Idee der „Optionszeiten“ (BMFSFJ 2006) - dem langfristigen Erhalt der Beschäftigungsfähigkeit und der besseren Vereinbarkeit der Lebensbereiche dienen, gilt unser Forschungsinteresse vorrangig den „optionalen Langzeitkonten“.

\section{Untersuchungsmethoden}

Die Projektergebnisse beruhen auf einem Methodenmix aus repräsentativen Unternehmensdaten, ausführlichen Betriebsfallstudien sowie quantitativen und qualitativen Beschäftigtenbefragungen:

(1) Repräsentativdaten über die Verbreitung und Ausrichtung von Langzeitkonten in den Unternehmen haben wir im Rahmen einer Unternehmensbefragung zu flexiblen Arbeitszeiten ausgewertet, die 2005 von der Sozialforschungsstelle Dortmund (Groß/ Schwarz 2007) erhoben worden sind.

(2) In acht Vorreiterbetrieben, die über ein Langzeitkonto verfügen, haben wir detaillierte Betriebsfallstudien durchgeführt. Bei den Betrieben handelt es sich vorrangig um Großbetriebe aus dem Industrie- und Dienstleistungssektor. Die Fallstudien basieren auf jeweils zehn bis 15 ausführlichen Expertengesprächen mit Vertretern der Unternehmensleitung und betrieblichen Interessenvertretung. Gegenstand der Gespräche waren die Motive und Strategien der Einführung des Langzeitkontos, bisherige Erfahrungen und Lernprozesse sowie mögliche Nutzungsbarrieren und Probleme in der betrieblichen Praxis.

(3) Durch standardisierte Beschäftigtenbefragungen in zwei der acht Betriebe - einem großen Dienstleistungsunternehmen und einem traditionellen Industriebetrieb - haben wir die Nutzungsinteressen und Nutzungsmuster männlicher und weiblicher Beschäftigter in unterschiedlichen Arbeits- und Lebenssituationen erfasst. Ergänzend dazu konnten wir in einem modernen Unternehmen aus dem Bereich der Informationstechnologie (IT) Personalund Zeiterfassungsdaten auswerten. Diese ermöglichen uns einen detaillierten Einblick in Unterschiede der Kontennutzung in Abhängigkeit von Geschlecht, Alter, Einkommen und Arbeitszeitsystem.

(4) Schließlich haben wir in fünf der Unternehmen insgesamt 25 qualitative Interviews mit unterschiedlichen Beschäftigtengruppen geführt und diese von ihren Motiven, Strategien, Erfahrungen und Schwierigkeiten bei der Nutzung des Langzeitkontos berichten lassen. Von besonderem Interesse für uns war dabei die Rolle des Langzeitkontos in den biografischen Entwürfen der Beschäftigten sowie dessen Zusammenspiel mit den Anforderungen der alltäglichen Lebensgestaltung.

In den folgenden vier Abschnitten werden die wichtigsten Befunde des Projekts in vier zentralen Untersuchungsfeldern dargestellt: Welche Motive und Strategien verfolgen die Betriebe bei der Einführung und Nutzung von Langzeitkonten (Abschnitt 3)? Welche Nutzungsmuster finden wir auf Seiten der Beschäftigten (Abschnitt 4)? Was sind die aktuellen Nutzungsbarrieren in der betrieblichen Praxis (Abschnitt 5)? Abschnitt 6 zieht Schlussfolgerungen für den arbeitspolitischen Handlungsbedarf.

\section{Einsatzstrategien der Unternehmen: Einseitige Ausrichtung des Langzeitkontos}

Unsere Auswertung der repräsentativen Unternehmensdaten belegt für Ende 2005, dass Langzeitkonten in $7 \%$ der deutschen Betriebe eingeführt sind. ${ }^{3}$ Der Anwendungsschwerpunkt der Konten liegt eindeutig bei den Großbetrieben. In dem Betriebssegment mit 500 oder mehr Beschäftigten finden wir bei fast jedem vierten Unternehmen ein Langzeitkonto. Eine überdurchschnittliche Verbreitung findet sich darüber hinaus in Betrieben mit einem hohen Flexibilitätsbedarf und mit einer längerfristig ausgerichteten Personalpolitik (Hildebrandt/ Wotschack 2006). Bei den angebotenen Verwendungszwecken gibt es Unterschiede nach der Betriebsgröße (Tabelle 1): Während in kleineren und mittleren Betrieben Optionen für temporäre Freistellungen („Familienzeit“ oder „temporäre Teilzeit") die häufigsten Nutzungskonstellationen sind, dominieren in den größeren Betrieben Möglichkeiten zur Verkürzung der Lebensarbeitszeit („,Vorruhestand“ oder „Altersteilzeit“), gefolgt von „beruflicher Weiterbildung“.

Die von uns durchgeführten ausführlichen Betriebsfallstudien machen deutlich, dass der Einsatz von Langzeitkonten überwiegend durch betriebliche Ziele wie Kapazitätsanpassung und Kostensenkung bestimmt wird, wobei jedoch zum Teil Spielraum für die Interessen der Beschäftigten gelassen wird. Die betrieblichen Ziele bei der Einführung von Langzeitkonten lassen sich auf drei Schwerpunkte fokussieren:

- Auffangen von Kapazitätsschwankungen und damit verbunden die Beschäftigungssicherung,

\footnotetext{
2 Das Projekt wurde unter dem Titel "Langzeitkonten und biografische Lebensführung " von der Hans-Böckler-Stiftung gefördert. An den Forschungsarbeiten waren neben den Verfassern beteiligt: Almut Kirschbaum, Svenja Pfahl.

3 Entsprechend unserer Definition handelt es sich dabei um Betriebe mit einem Arbeitszeitkonto, das einen Ausgleichszeitraum länger als ein Jahr aufweist. Wird eine engere Definition von Langzeitkonten zugrunde gelegt (Existenz eines "separaten Langzeitkontos"), fällt die Verbreitung mit $2 \%$ deutlich geringer aus (Fischer et al. 2007).
} 


\begin{tabular}{|c|c|c|c|c|c|}
\hline & $\begin{array}{c}\text { Alle } \\
\text { Betriebe }\end{array}$ & $\begin{array}{c}1-9 \\
\text { Beschäftigte }\end{array}$ & $\begin{array}{c}10-49 \\
\text { Beschäftigte }\end{array}$ & $\begin{array}{c}\text { 50-249 } \\
\text { Beschäftigte }\end{array}$ & $\begin{array}{c}250+ \\
\text { Beschäftigte }\end{array}$ \\
\hline Weiterbildung & 17 & 17 & 12 & 27 & 50 \\
\hline Sabbatical & 6 & 2 & 9 & 17 & 27 \\
\hline Familienzeit & 27 & 17 & 39 & 42 & 26 \\
\hline Temporäre Teilzeit & 30 & 17 & 45 & 47 & 28 \\
\hline Altersteilzeit & 7 & 6 & 1 & 23 & 69 \\
\hline Vorruhestand & 6 & 6 & 1 & 20 & 54 \\
\hline Sonstiges & 64 & 64 & 70 & 51 & 34 \\
\hline \multicolumn{6}{|c|}{ Nur Betriebe mit Langzeitkonto: $\mathrm{n}=204$. } \\
\hline $\begin{array}{l}\text { Quelle: Auswertungen } \\
\text { durch das WZB. }\end{array}$ & ativen Bet & agung der & rtmund (2005) & VSII & TTEILUNGEN \\
\hline
\end{tabular}

- Ermöglichung von Zeitoptionen für die Mitarbeiter und

- Aufbau einer neuen Altersübergangsregelung.

Die Dominanz betrieblicher Interessen zeigt sich auch in der Ausgestaltung der Konzipierungs- und Einführungsprozesse der Konten. Nur in Ausnahmen bestand ein wirkliches betriebliches Interesse daran, die zeitlichen Interessenlagen und Handlungsmuster der Beschäftigten bezüglich des Langzeitkontos zu ermitteln und einzubeziehen. Nur vereinzelt gab es in den von uns untersuchten Betrieben vorbereitende Beschäftigtenbefragungen, Gruppengespräche oder eine direkte Einbeziehung der Mitarbeiter in die Konzipierung. Das ist bei einem derart komplexen Arbeitszeitinstrument, bei dem es um langfristige, größere Zeitinvestitionen geht, die die alltägliche und biografische Lebensgestaltung der Beschäftigten tangieren, erstaunlich. Die Beteiligung der betrieblichen Interessenvertretungen an den Einführungsprozessen, zum Teil auch unter Einbezug der Gewerkschaften, war allerdings durchgängig hoch. In zwei Fällen ging die Einführung sogar auf eine Initiative des Betriebsrats zurück. In allen von uns untersuchten Betrieben gab es gemeinsame Beratungen und Regelungen; oft wurde eine gemeinsame Konflikt-Kommission eingesetzt, die auf die regelgerechte Handhabung der Konten achtet.

Bei den angebotenen Verwendungszwecken zeigt sich ein klarer Trend zur Schwerpunktsetzung oder (zusätzlichen) Einbeziehung von Angeboten, die sich auf den Altersübergang beziehen. Auch die fehlende Abstimmung und Verknüpfung der Nutzungsmöglichkeiten des Langzeitkontos mit Human-Ressources (HR)-Maßnahmen in den Bereichen Familie, Weiterbildung und Gesundheit deuten auf eine eher einseitige Ausrichtung des Langzeitkontos hin: Obwohl es sich bei den von uns untersuchten Betrieben überwiegend um Großbetriebe in guter wirtschaftlicher Lage handelt, die in den genannten Feldern der Arbeitspolitik über strukturierte Programme verfügen, wurde die Verbindung $\mathrm{zu}$ Langzeitkonten programmatisch und regelungstechnisch nicht vollzogen. Zugespitzt ließe sich sagen, dass die Langzeitkonten nicht in die betriebliche Pflege der Humanressourcen einbezogen werden. Vielmehr werden die angelegten Optionsmöglichkeiten (alltäglich und mittelfristig) der individuellen Verantwortung der Beschäftigten zugeordnet. Die Optionen im Rahmen von Langzeitkonten und ihre Realisierung sind, was ihre Verwendung angeht, Sache des Einzelnen und nicht des Betriebs.

\section{Geringe Resonanz auf Seiten der Beschäftigten: fünf Ursachen}

Auf Seiten der Beschäftigten ist eine zurückhaltende Nutzung des Langzeitkontos zu beobachten. Bei den von uns befragten Beschäftigten eines (mittelgroßen) Industriebetriebs befürwortete zum Zeitpunkt der Befragung nur jeder Vierte eine Ausweitung des bestehenden Arbeitszeitkontensystems zum Langzeitkonto; auch unter den befragten Beschäftigten eines großen Dienstleistungsunternehmens nutzt nur jeder Vierte das Langzeitkonto. Dieser Grundeindruck wird auch durch die anderen von uns untersuchten Betriebsfälle gestützt. Von wenigen Ausnahmen abgesehen, stellen sich die Akzeptanz und aktive Nutzung des Langzeitkontos durch die Beschäftigten in den Betrieben als problema- tisch dar. Die Beschäftigtenbefragungen lassen ein grundlegendes Spannungsverhältnis zwischen Bedarf und Nutzung erkennen: Auf der einen Seite zeigt sich ein hoher zeitlicher Bedarf, den Alltag zu entlasten, die Erwerbsdauer zu verkürzen und neue Unsicherheiten des Erwerbsverlaufs zu bewältigen. Auf der anderen Seite wird das langfristige Ansparen von Mehrarbeit auf einem Langzeitkonto von der Mehrheit der Beschäftigten nicht als attraktives bzw. geeignetes Instrument wahrgenommen, um derartigen Anforderungen oder Wünschen Rechnung zu tragen. Insgesamt zeigt sich, dass oft gerade die Gruppen, die am dringlichsten auf zeitliche Entlastung angewiesen sind (etwa Beschäftigte mit Kindern oder Pflegeaufgaben), kaum Guthaben mit dem Langzeitkonto aufbauen können. Dieser Grundwiderspruch stellt eine zentrale Hürde für die Entfaltung des Potenzials von Langzeitkonten dar. Als verantwortlich dafür konnten wir in unserer Untersuchung fünf zentrale Ursachenkomplexe identifizieren.

\subsection{HOHER ZEIT- UND GELDBEDARF VOR ALLEM IN DER MITTLEREN LEBENSPHASE}

Der Erhalt der alltäglichen Balance von Arbeit und Privatleben (inklusive ausreichendem Haushaltseinkommen) lässt für viele Beschäftigte wenig Spielraum für die Nutzung des Langzeitkontos. Unsere (quantitativen und qualitativen) Beschäftigtenbefragungen belegen eindrucksvoll den hohen Stellenwert, den die tägliche Balance von beruflichen und außerberuflichen Aktivitäten für die Mehrheit der Beschäftigten hat (Abbildung 1). Das gilt für männliche wie für weibliche Beschäftigte, im Angestelltenbereich wie im gewerblichen oder operativen Bereich sowie für Beschäftigte in verschiedenen Lebensphasen. Die tägliche Balance von Beruf und Privatleben wird auf der einen Seite durch den hohen betrieblichen Flexibilitäts- und Mehrarbeitsbedarf stark unter Druck gesetzt, auf der anderen Seite durch private Zeit- und Geldbedarfe der Beschäftigten, die vor allem in der Familienphase unabweisbar sind (vgl. auch Schier/Jurczyk 2007; DGB-Index Gute Arbeit 2007). In der Folge sind Möglichkeiten zum kurzfristigen Zeit- und finanziellen Ausgleich von Mehrarbeit für viele Beschäftigte substanziell, um die tägliche Balance zwischen den verschiedenen Lebensbereichen $\mathrm{zu}$ wahren oder sie zu- 
rückzugewinnen. Besonders hohe Geldund Zeitbedarfe zeigen sich abhängig von der Lebensphase und der jeweiligen Erwerbs- und Familienkonstellation. Sie sind bei Beschäftigten in der mittleren Lebensphase ("Rushhour of Life") besonders hoch. Auch in traditionellen Haushaltskonstellationen (männliches Ernährermodell) und bei eher niedrigem Einkommen entstehen zeitliche Engpässe für die Beschäftigten durch das Zusammenfallen von Ernährerrolle und Familienphase. In Zweiverdienerhaushalten (insbesondere wenn beide Partner Vollzeit arbeiten) wird die Vereinbarkeit zusätzlich durch konkurrierende berufliche und außerberufliche Anforderungen erschwert (Solga/Wimbauer 2005).

\subsection{KAUM INTERESSE AN LANG- FRISTIGEN ZEITSPAROPTIONEN}

Obwohl der Ausstieg aus dem Erwerbsleben vor der gesetzlichen Rentengrenze ein weitverbreitetes Interesse der von uns befragten Beschäftigten ist, stellen langfristige Zeitsparmodelle für die wenigsten eine attraktive Option dar, um einen solchen Ausstieg zu realisieren. Insbesondere die hohen Arbeitsbelastungen bzw. die damit verbundenen gesundheitlichen Beeinträchtigungen, die wir bei Angestellten wie Gewerblichen finden, tragen dazu bei, dass fast alle der von uns befragten Beschäftigten angeben, vor dem gesetzlichen Rentenalter in den Ruhestand gehen zu wollen, auch wenn damit finanzielle Einbußen verbunden sind; die Mehrheit gibt sogar an, höchstens bis zum 60. Lebensjahr erwerbstätig sein zu wollen. Trotz dieser klaren Orientierung auf ein vorzeitiges Ausscheiden aus dem Erwerbsleben äußert in den quantitativen Beschäftigtenbefragungen nur eine kleine Minderheit Interesse und Bereitschaft, für diesen Zweck langfristig Mehrarbeit anzusparen. Die qualitativen Interviews machen deutlich, dass sich eine Reihe von Beschäftigten zwar durchaus dafür interessiert, für einen früheren Altersübergang Zeit anzusparen, dies aber unter den gegebenen Alltagsanforderungen schlichtweg für nicht leistbar hält. Das Langzeitkonto wird daher von der Mehrheit der Beschäftigten nicht als geeignetes Instrument zur Realisierung dieses Ziels angesehen. Bevorzugt werden demgegenüber eher private Geldanlagen, auch um mögliche Rentenabschläge bei vorgezogenem Altersübergang auszugleichen.

\section{Abb. 1: Bevorzugte Zeiteinteilung von männlichen und weiblichen}

Beschäftigten* - in \% -

Welche persönliche Zeiteinteilung bevorzugen Sie?

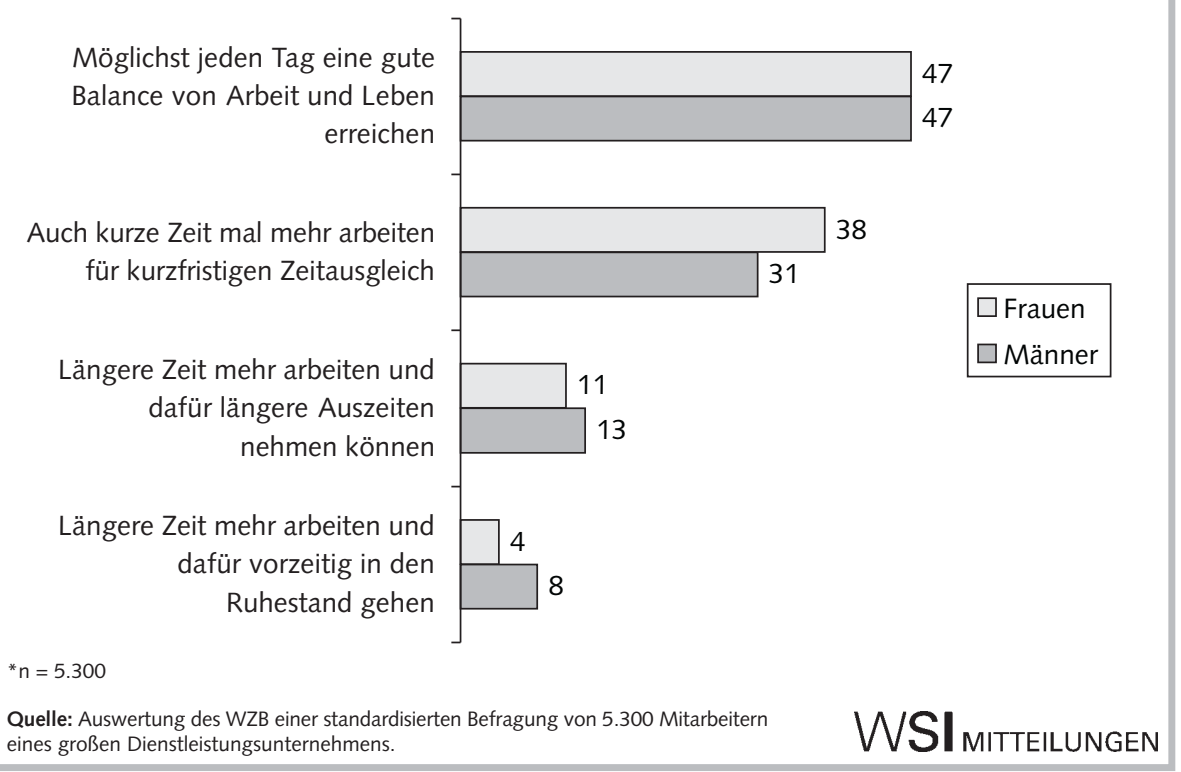

\subsection{HOHE ZUKUNFTSUNSICHERHEIT}

Der Nutzen langfristig angelegter Arbeitszeitinstrumente setzt einen ebenso langfristigen Verbleib im Unternehmen voraus. So erstaunt es dann auch nicht, dass die Erfahrung beruflicher Unsicherheiten oder eine fehlende Langfristperspektive im Unternehmen sich nachteilig auf die Nutzung des Langzeitkontos auswirken. In fast allen Befragungsbetrieben haben umfangreiche Entlassungen stattgefunden, die in der Regel zu einer großen Verunsicherung der Beschäftigten hinsichtlich ihrer langfristigen Beschäftigungssicherheit und beruflichen Perspektive im Unternehmen führen. Von den befragten Beschäftigten des (mittelgroßen) Industriebetriebes gibt jeder Zweite an, dass es große Unsicherheiten im privaten oder beruflichen Bereich gäbe; bei den Beschäftigten des großen Dienstleistungsunternehmens hat nur jeder Zweite ein klares Gefühl von Arbeitsplatzsicherheit; nur jeder Vierte sieht für sich gute berufliche Entwicklungsmöglichkeiten im Unternehmen. Dieses gravierende Bewusstsein von Zukunftsunsicherheit führt nur bei wenigen dazu, das Langzeitkonto als ein mögliches Instrument, als Sicherheitspolster für eine berufliche Umorientierung oder einen drohendem Arbeitsplatzverlust zu nutzen. Bei der Mehrheit begrenzt die fehlende Langfristperspektive im Unternehmen eher die Bereitschaft, sich auf ein auf die weitere Zukunft orientiertes Instru- ment wie das Langzeitkonto einzulassen. Viele sorgen sich auch um einen möglichen Verfall des gesparten Zeitguthabens oder bezweifeln den persönlichen Nutzen der erst Jahre später zu entnehmenden Zeitguthaben.

\subsection{KURZFRISTIGER PLANUNGSHORIZONT}

Bei der Mehrheit der von uns befragten Beschäftigten dominieren eine kurz- bis mittelfristige biografische Perspektive und eher zeitnahe Verwendungsinteressen. Langfristig angelegte biografische Entwürfe und Strategien, die einen Zeitraum von fünf Jahren überschreiten, finden wir kaum. Die starke Orientierung auf die alltägliche Balance und der große Einfluss der mit der Arbeits- und Lebenssituation gegebenen Zeit- und Geldbedarfe spiegeln sich deutlich in den Verwendungsinteressen der Beschäftigten wider. Die standardisierten Beschäftigtenbefragungen zeigen, dass bei den Nutzern des Langzeitkontos ein vorzeitiges Ausscheiden aus dem Erwerbsleben sowie Auszeiten für die Familie zu den am meisten gewünschten Verwendungen gehören (Abbildung 2). Weiterbildung, vorübergehende Teilzeit und längere Auszeiten für persönliche Interessen (Sabbaticals) spielen eine deutlich geringere Rolle. In dem von uns untersuchten Industriebetrieb, in dem die gesundheitlichen Belastungen besonders hoch sind, hat Zeit für 


\section{Abb. 2: Gewünschte Verwendung des Zeitguthabens von Nutzern des Langzeitkontos nach Geschlecht* - in \% -}

Welche Verwendung der angesparten Zeit bevorzugen Sie?

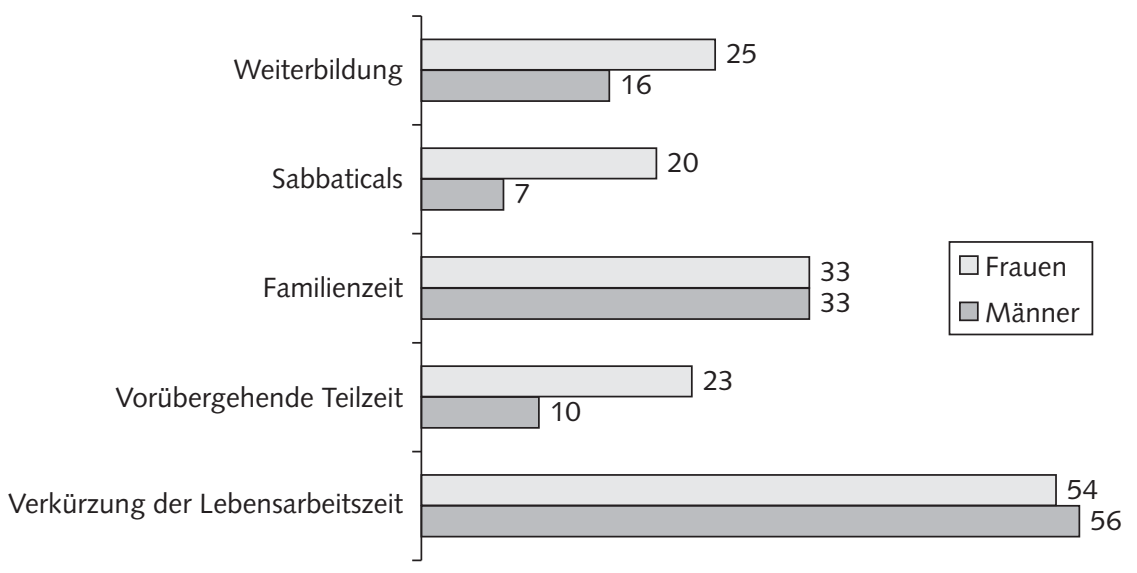

${ }^{*} \mathrm{n}=5.300$

Quelle: Auswertung des WZB einer standardisierten Befragung von 5.300 Mitarbeitern eines großen Dienstleistungsunternehmens.

WSI MITTELUNGEN

\section{Abb. 3: Nutzung des Langzeitkontos nach Geschlecht und Unternehmensbereich* - in \% -}

Nutzen Sie das Langzeitkonto?

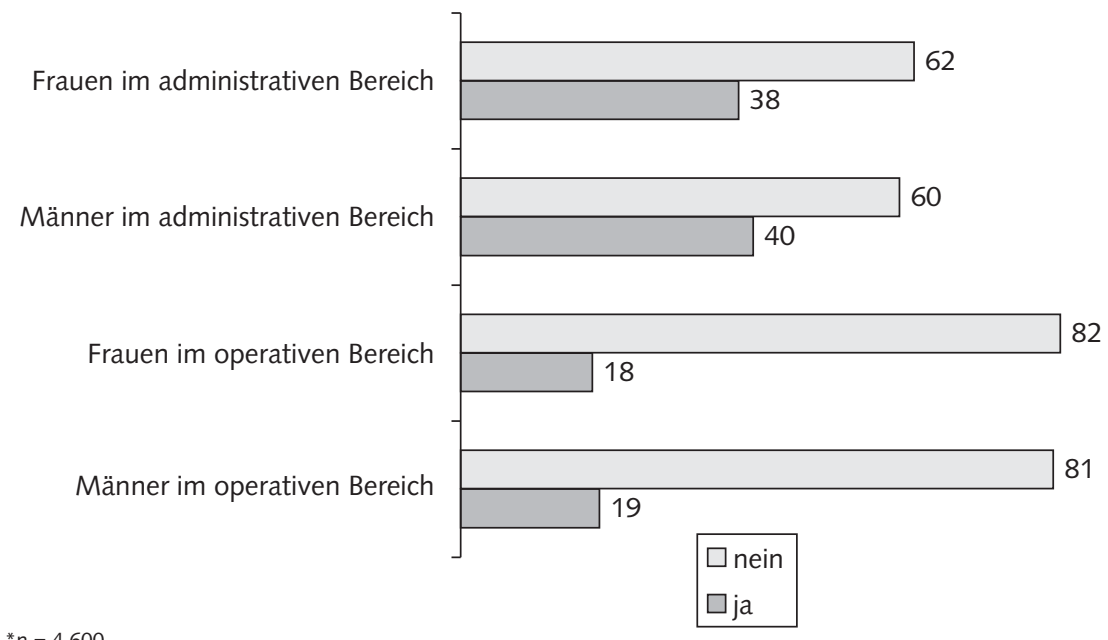

${ }^{*} \mathrm{n}=4.600$

Quelle: Auswertung des WZB einer standardisierten Befragung von 5.300 Mitarbeitern eines großen Dienstleistungsunternehmens.

\section{WSI MITTELUNGeN}

Erholung und Gesundheit eine große Bedeutung. Insgesamt sind die Verwendungswünsche eher zeitnah, auf die aktuelle Lebensphase oder die nahe Zukunft ausgerichtet. Jüngere Beschäftigte (vor der Familienphase) und Beschäftigte mit Kindern wünschen sich häufiger Familienzeit; ältere Beschäftigte einen vorzeitigen oder gleitenden Ruhestand. Eine langfristige Orientierung und Lebensplanung ist in den Verwendungswünschen kaum zu erkenterviews zeigt sich deutlich, dass Langzeitkonten in keinem Fall konstituierend oder Anstoß für eine stärker biografisch orientierte Lebensführung waren. Allerdings finden wir auch Hinweise darauf, dass mit dem Anwachsen größerer Zeitguthaben erste Such- und Lernprozesse bezüglich deren möglicher Verwendung im Rahmen der biografischen Lebensgestaltung in Gang gesetzt werden.

\subsection{GERINGE RESONANZ IM GEWERBLICHEN UND OPERATIVEN BEREICH}

Ein klares Nutzerprofil bezüglich des Langzeitkontos lässt unsere Untersuchung zwar (noch) nicht erkennen. Unterschiede zwischen männlichen und weiblichen Beschäftigten sind in der Verbreitung des Langzeitkontos nicht auszumachen; in den Verwendungsinteressen sind sie eher schwach ausgeprägt. Zugleich belegt unsere Untersuchung, dass Unterschieden nach der beruflichen Stellung und Qualifikation bei der Verbreitung des Langzeitkontos eine große Rolle zukommt. Das Interesse am Langzeitkonto ist bei den befragten Beschäftigten im gewerblichen und operativen Bereich am niedrigsten; bei den meist höher qualifizierten Dienstleistungsbeschäftigten ist die Verbreitung des Langzeitkontos hingegen doppelt so hoch ( $A b$ bildung 3). Eine Vorreiterrolle in unserer Untersuchung nehmen die hoch qualifizierten Beschäftigten eines IT-Unternehmens ein. In diesem Betrieb haben vier von fünf Beschäftigten ein Guthaben auf dem Langzeitkonto. Das durchschnittliche Zeitguthaben betrug sieben Jahre nach der Einführung des Langzeitkontos (2000) 170 Stunden; bei älteren Beschäftigten (über 55 Jahre) und bei den Spitzenverdienern fiel es erheblich größer aus. Die Vorreiterrolle lässt sich zum einen durch das insgesamt höhere Mehrarbeitsvolumen und Einkommensniveau erklären, dass für höher qualifizierte bzw. hoch qualifizierte Angestellte typisch ist. Bei den Beschäftigten des ITUnternehmens spielt darüber hinaus die Möglichkeit eine entscheidende Rolle, auch Entgeltbestandteile ins Langzeitkonto einbringen zu können. Gleichzeitig wirken sich bei dieser Gruppe schließlich auch die mit dem Berufsfeld (hoch qualifizierte wissensbasierte Tätigkeiten) verbundenen hohen Kompetenzen in Bezug auf individuelle Planung, Kalkulation und selbstständige Steuerung der Arbeitszeiten positiv auf die Nutzung des Langzeitkontos aus.
Barrieren in der betrieblichen Praxis

Langzeitkonten sind ein Baustein einer langfristig angelegten Unternehmenspolitik. Sie gehen - insbesondere solange keine 
Regelungen zur Portabilität existieren von einer langfristigen Betriebszugehörigkeit der Beschäftigten aus, gegebenenfalls bis zum Ende des Erwerbslebens. Dieses Konstruktionsprinzip gerät zunehmend in Widerspruch zur generellen Flexibilisierung der Beschäftigungsverhältnisse (Keller/Seifert 2007). Bei Langzeitkonten handelt es sich faktisch um einen Langfristkredit der Beschäftigten an das Unternehmen (mit Varianzen nach Verzinsung und Anlageform) und damit um ein neues, höchst ungewöhnliches Geschäft zwischen Unternehmen und Beschäftigten. Diese Tauschbeziehung wird in dem gleichen Maße problematisch, in dem der zukünftige Ausgleich und die gewünschte Verwendung des Zeitguthabens unsicher werden. In unserer Untersuchung konnten wir eine Reihe betrieblicher Faktoren identifizieren, die sich hier hemmend auswirken.

\subsection{ARBEITSPLATZRISIKO}

Für Beschäftigte in atypischer, befristeter oder unsicherer Beschäftigung ist der Nutzen einer Investition in langfristig angelegte betriebliche Instrumente eher fraglich. Im Falle der Insolvenz, des Arbeitsplatzverlustes oder eines Arbeitgeberwechsels (sogenannte „Störfälle“) kann das Zeitguthaben bisher nicht übertragen werden (Portabilität). Durch Insolvenzschutz (der aber immer noch nicht überall eine Selbstverständlichkeit ist) kann der Verlust des Guthabens zwar verhindert werden. Der eigentliche Nutzen des Instruments, in Form einer längeren bezahlten Freistellung, wird damit allerdings verfehlt. Dieser entsteht erst durch den langfristigen Aufbau von Guthaben und setzt eine langfristige Beschäftigungs- und Karriereperspektive im Unternehmen voraus. In den von uns untersuchten Betrieben mit Langzeitkonto finden wir folglich auf der einen Seite einen durchgängigen Ausschluss der Randbelegschaften von der Kontenregelung. Darüber hinaus zeigt sich, dass Beschäftigte, die mit besonderen beruflichen Unsicherheiten konfrontiert sind, seltener das Langzeitkonto nutzen.

\subsection{GESTÖRTE VERTRAUENS- BEZIEHUNGEN}

Die bisherigen Erfahrungen der Beschäftigten mit der Arbeitszeitpraxis und Personalpolitik des Unternehmens stellen eine wichtige Einflussgröße für die Akzeptanz des Instruments der Langzeitkonten dar. Beschäftige, die in der Vergangenheit einen ausgewogenen Ausgleich von Unternehmens- und Mitarbeiterinteressen erlebt haben, die Vertrauen in die Leitungs- und Führungsebene haben und für sich Sicherheit und berufliche Entwicklungsmöglichkeiten im Unternehmen sehen, sind auch eher bereit, sich auf ein Langzeitkonto einzulassen. In vielen der von uns befragten Betriebe zeichnen die Beschäftigten allerdings ein eher kritisches Bild der Arbeitszeitpraxis und Personalpolitik. Sind die Reziprozitäts- und Vertrauensbeziehungen zwischen Betrieb und Beschäftigten gestört, sinkt die Bereitschaft für langfristige Zeit- oder Geldinvestitionen in das Langzeitkonto. In diesem Zusammenhang wirken sich auch die Barrieren bei optionalen Zeitentnahmen vom Langzeitkonto aus (Zeitpunkt, Umfang, Verwendung des Zeitguthabens), die im Betrieb kommuniziert und dementsprechend von vielen Beschäftigten schon im Vorfeld antizipiert werden. Restriktionen, die durch die Arbeitsorganisation, Vorgesetzte oder mögliche Nachteile für die Karrierechancen entstehen, fließen bei der Einschätzung des Instruments durch die Beschäftigten mit ein.

\subsection{FEHLENDE FLANKIERUNG DURCH DIE PERSONALPOLITIK}

Die Akzeptanz des Langzeitkontos wächst mit dessen Einbindung und Unterstützung durch die Personalpolitik. Die Art der Kapazitätsplanung und -steuerung, das Verhalten der Vorgesetzten, die Leistungspolitik und die betriebliche Zeitkultur sowie unterstützende Information und Kommunikation beeinflussen die Anspar- und Entnahmeprozesse maßgeblich. Da in den von uns untersuchten Betrieben von der Leitungsebene kaum starke Signale zur Unterstützung der Langzeitkonten oder bestimmter Entnahmezwecke ausgesendet werden, ist der Einfluss der direkten Vorgesetzten sehr groß. Häufig sind sie nicht extra angewiesen und geschult worden, sondern verfahren nach persönlichen Präferenzen und dem akuten Bedarf. In der Folge sind größere Entnahmen kaum möglich. Da der Stellenwert der Langzeitkonten in der Personal-, Leistungs- und Arbeitszeitpolitik relativ gering ist, werden die genannten Einflussfaktoren nur selten auf die Unterstützung der Langzeitkonten eingestellt und wirken sich daher häufig hemmend oder widersprüchlich aus. Der Auf- bau von entsprechenden Kompetenzen und die systematische Unterstützung von Lernprozessen als wichtige Voraussetzungen für ein erweitertes biografisches Handeln werden vom Betrieb nicht gefördert.

\section{4 ÜBERFORDERUNG DES INSTRUMENTS}

Wenn Langzeitkonten sowohl auf den vorzeitigen Ausstieg aus dem Erwerbsleben als auch auf präventive Maßnahmen zur Erhöhung der langfristigen Beschäftigungsfähigkeit ausgerichtet werden, findet eine Überforderung des Instruments statt. Eine einfache Modellrechnung macht die begrenzten Ansparmöglichkeiten mit dem Langzeitkonto deutlich: Wenn jedes Jahr wöchentlich zwei Stunden Mehrarbeit in das Langzeitkonto eingebracht werden und man von 220 Arbeitstagen pro Jahr ausgeht, dauert es ca. 22 Jahre, bis eine Freistellung im Umfang eines Jahres angespart ist. Damit wird klar, dass viele Beschäftigte das Langzeitkonto nicht für mehrere Zwecke nutzen können. Es entsteht eine Konkurrenz zwischen unterschiedlichen Verwendungsmöglichkeiten, vor allem zwischen befristeten, optionalen Freistellungen während der Erwerbstätigkeit einerseits und zusätzlicher finanzieller Altersvorsorge und Verkürzung der Lebensarbeitszeit andererseits. Dieser Konflikt wird zum Teil durch die Öffnung für Einstellungsmöglichkeiten in Geld gemildert. Insbesondere aufgrund der Langfristigkeit der erforderlichen Ansparprozesse und des erforderlichen Volumens bleibt allerdings fraglich, ob Langzeitkonten als Auffanglösung für Frühverrentung, Altersteilzeit und Rentenniveauabsenkung geeignet sind. Schon jetzt lässt sich sagen, dass diese Inanspruchnahme die Zeit- und Geldkonten quantitativ überfordert. Es besteht zumindest die Gefahr, dass Optionen, die dem Erhalt der Beschäftigungsfähigkeit und der besseren Vereinbarkeit der Lebensbereiche dienen, den Ansparerfordernissen für den Altersübergang untergeordnet werden.

\section{Fazit und Ausblick}

\subsection{KAUM AUSSCHÖPFUNG DES POTENZIALS VON LANGZEITKONTEN}

Vor dem Hintergrund der skizzierten Befunde zeigt sich, dass ein Potenzial von 
Langzeitkonten für eine bessere Zeit- und Geldverteilung im Lebenslauf zwar zu erkennen ist, sich in der aktuellen betrieblichen Praxis aber höchstens ansatzweise entfaltet. Mit Blick auf die Alltagsanforderungen und Verwendungsinteressen zeigt sich bei männlichen und weiblichen Beschäftigten ein hoher Zeitbedarf für Familie, Kinderbetreuung, Pflege und Erholung. Daneben steht der Wunsch nach vorzeitigem oder gleitendem Ruhestand oder der Absicherung von privaten und beruflichen Risiken und Übergängen. Die insgesamt eher geringe Resonanz auf das Langzeitkonto als ein mögliches Instrument, um diese Bedarfe zu realisieren, ist in erster Linie auf Restriktionen im Anspar- und Entnahmeprozess zurückzuführen, die sich einerseits aus betrieblichen Faktoren ergeben (etwa dem Kontensystem oder der gegebenen Arbeitszeitpraxis), zum anderen durch Zwänge der Einkommens-, Arbeits- und Lebenssituation der Beschäftigten. Diese Restriktionen sind derzeit nicht nur für die insgesamt geringe Nutzung des Langzeitkontos verantwortlich, sie begründen auch auf Seiten der Beschäftigten unterschiedliche Nutzungsmuster und erhöhen damit die Gefahr der Selektivität des Instruments. Auch wenn wir bisher wenig über die Entnahmepraxis des Langzeitkontos wissen, besteht Grund zu der Annahme, dass sich betriebliche und außerberufliche Restriktionen auch dort fortsetzen und den Einfluss der Beschäftigten auf Zeitpunkt, Umfang und Verwendung der entnommen Zeit begrenzen werden.

\subsection{AUFGABEN UND HANDLUNGS- FELDER DER ARBEITSPOLITIK}

Aus den skizzierten Schwierigkeiten lassen sich Aufgaben und Handlungsfelder für die betriebliche und überbetriebliche Arbeitspolitik ableiten:
(1) Den Erfordernissen der alltäglichen Balance der Beschäftigten ist verstärkt Rechnung zu tragen. Konkret heißt das, dass Langzeitkonten von Kurzzeitkonten (Gleitzeit- oder Jahresarbeitszeitkonten) zu trennen sind. Auch die Übertragbarkeit von Mehrarbeit von Kurz- auf Langzeitkonten wäre zu begrenzen. Auf diese Weise wird eine gezielte Steuerung der Schnittstelle zwischen alltäglicher und biografischer Lebensgestaltung möglich.

(2) Konfliktkommissionen haben sich in der Praxis bewährt. Sie können den Einsatz von Langzeitkonten anhand von Beschäftigtenbefragungen, regelmäßigem Informationsaustausch und Hinweisen auf Nutzungsprobleme verbessern. Um darüber hinaus Lernprozesse systematisch zu unterstützen, sind Beratungs- und Schulungsstellen erforderlich.

(3) Um Barrieren bei der Entnahme zu verringern, wären Regelungen mit möglichst verbindlichen Ansprüchen und Rückkehrgarantien zu formulieren. Eine Optimierung der betrieblichen Kapazitätssteuerung kann darüber hinaus eine bessere Organisation und Planung von Freistellungen ermöglichen.

(4) Um die Zugangs- und Ansparmöglichkeiten zu verbessern, erscheint eine stärkere Flankierung und Unterstützung von Langzeitkonten durch Staat und Arbeitgeber erforderlich. Gleichzeitig ist eine Verbesserung des Insolvenzschutzes zu gewährleisten und über Möglichkeiten der Portabilität nachzudenken (vgl. zu diesbezüglichen Ansätzen in den Niederlanden Wotschack 2007). Zu diskutieren wären auch neuartige Verknüpfungen mit staatlichen Sicherungssystemen, wie sie etwa das Konzept der „Arbeitslebensversicherung“ (Schmid 2004) vorschlägt.
(5) Die Altersteilzeit ist neu zu regeln mit dem Ziel, Eingliederung statt Ausgliederung zu erreichen. Auf Basis einer wirklichen Teilzeitbeschäftigung (Teilzeit im Alter) kann einerseits ein gleitender Übergang von Erwerbsarbeit in den Ruhestand erfolgen, andererseits der Einstieg in Pflege, Ehrenamt und Weiterbildung.

(6) Schließlich erscheint eine systematische Einbettung und Verknüpfung von Langzeitkonten mit anderen Feldern der Arbeitspolitik sinnvoll. Durch die Verbindung des Langzeitkontos mit konkreten Angeboten und Programmen der betrieblichen Weiterbildung, Gesundheitsprävention oder im Bereich der Vereinbarkeit von Beruf und Familie sind Synergien und wichtige Impulse für eine nachhaltige Personalpolitik zu erwarten.

Insbesondere zu dem letzten Punkt wäre auch zu untersuchen, welche neuen betrieblichen Handlungsansätze derzeit in den Unternehmen unter dem Stichwort "Gestaltung des demografischen Wandels“ oder „Lebenszyklusorientierte Personalarbeit" entwickelt werden (IG-Metall Vorstand 2007; Econsene 2006).

Langzeitkonten entfalten nicht automatisch ihr Potenzial für erweiterte Arbeitszeitoptionen und neue Lebensläufe. Sie sind auch nicht für alle Beschäftigtengruppen gleichermaßen zugänglich und nutzbar. Und sie stellen kein universales Allheilmittel für die demografischen $\mathrm{He}$ rausforderungen dar. Langzeitkonten können aber in all diesen Bereichen einen wichtigen Beitrag leisten. Ohne eine entsprechende Verbindung mit anderen Feldern und Instrumenten der Arbeitspolitik und ohne die gezielte betriebliche und überbetriebliche Regulierung und Flankierung wird dieser Beitrag allerdings gering ausfallen. 
Anxo, D./Erhel, Ch./Schippers, J. (Hrsg.) (2008): Labour Market Transitions and Time Adjustment over the Life Course, Amsterdam Brandl, S./Hildebrandt, E./Wotschack, P. (Hrsg.) (2008): Arbeitszeitpolitik im Lebensverlauf. Ambivalenzen und Gestaltungsoptionen in deutscher und europäischer Perspektive. Edition der Hans-Böckler-Stiftung, Düsseldorf (im Erscheinen)

Brinkmann, U./Dörre, K./Röbenack, S./Kraemer, K./Speidel, F. (2006):

Prekäre Arbeit - Ursachen, Ausmaß, soziale Folgen und subjektive Verarbeitungsformen unsicherer Beschäftigungsverhältnisse, Studie im Auftrag der Friedrich-Ebert Stiftung, Bonn

Bundesministerium für Familie, Senioren, Frauen und Jugend (BMFSFJ) (2006): Siebter Familienbericht. Familie zwischen Flexibilität und Verlässlichkeit - Perspektiven für eine lebenslaufbezogene Familienpolitik, Berlin Deutscher Bundestag (2002): Schlussbericht der Enquete-Kommission „Demographischer Wandel - Herausforderungen unserer älter werdenden Gesellschaft an den Einzelnen und die Politik“" 14. Wahlperiode, Drucksache 14/8800, 28.03.

DGB-Index Gute Arbeit (2007): Work-Life-Balance 2007 - Der Report. Wie die Beschäftigten die Vereinbarkeit von Berufs-, Familien- und Privatleben beurteilen, Berlin

Deutsche Industrie- und Handelskammer (DIHK) (2004): Individuell und flexibel. Wettbewerbsfaktor Arbeitszeitgestaltung. Ergebnisse einer DIHKUnternehmensbefragung, Berlin

Econsense (2006): Die demographische Herausforderung gestalten, Arbeitsdokument, Econsense - Forum Nachhaltige Entwicklung der Deutschen Wirtschaft e. V., Berlin

European Commission (2005): Confronting demographic change: a new solidarity between the generations. 16.03., COM (2005) 94 final, Brüssel Fischer, G./Wahse, J./Dahms, V./Frei, M./Riedmann, A./Janik, F. (2007): Standortbedingungen und Beschäftigung in den Regionen West- und Ostdeutschlands. Ergebnisse des IAB-Betriebspanels 2006, in: IAB-Forschungsbericht 5, Nürnberg

Groß, H./Schwarz, M. (2007): Betriebs- und Arbeitszeiten 2005. Ergebnisse einer repräsentativen Betriebsbefragung. Sozialforschungsstelle Dortmund (sfs), Beiträge aus der Forschung, Band 153, Dortmund Groß, H./Seifert, H./Sieglen, G. (2007): Formen und Ausmaß verstärkter Arbeitszeitflexibilisierung, in: WSI-Mitteilungen 4, S. 202-208
Hildebrandt, E. (Hrsg.) (2007): Lebenslaufpolitik im Betrieb. Optionen zur Gestaltung der Lebensarbeitszeit durch Langzeitkonten, Berlin Hildebrandt, E./Wotschack, P. (2006): Langzeitkonten und Lebenslaufpolitik, in: WSI-Miteilungen 11, S. 592-600

Hildebrandt, E./Wotschack, P./Kirschbaum, A. unter Mitarbeit von Pfahl, S./Scheier, F. (2008): Endbericht des Projekts „Langzeitkonten und biographische Lebensführung “, Hans-Böckler-Stiftung, Düsseldorf IG-Metall Vorstand (2007): Recherchebericht 2007. Betriebliche Handlungsansätze zur Gestaltung des demographischen Wandels in zehn Industrieunternehmen, Hamburg

Institut der Deutschen Wirtschaft (IW) (2006): Projektergebnisbericht: Lebenszyklusorientierte Personalpolitik - „Work-Life-Balance“-Modelle und "Demographietools" für die betriebliche Praxis, Studie des Instituts der deutschen Wirtschaft im Auftrag der DekaBank, Köln

Keller, B./Seifert, H. (Hrsg.) (2007): Atypische Beschäftigung - Flexibilisierung und soziale Risiken, Berlin

Schier, M./Jurczyk, K. (2007): Familie als Herstellungsleistung in Zeiten der Entgrenzung, in: Aus Politik und Zeitgeschichte 34, S. 10-17 Schmid, G. (2004): Risikomanagement im europäischen Sozialmodell. Arbeitsmarktpolitische und normative Aspekte eines Paradigmenwechsels, in: Kaelbe, H./Schmid, G. (Hrsg.): Das europäische Sozialmodell. Auf dem Weg zum transnationalen Sozialstaat, WZB-Jahrbuch 2004, Berlin, S. 375-421

Seifert, H. (2005): Vom Gleitzeit- zum Langzeitkonto, in: WSI-Mitteilungen 6, S. 308-313

Solga, H./Wimbauer, C.. (2005): "Wenn zwei das Gleiche tun ..." Ideal und Realität sozialer (Un-)Gleichheit in Dual Career Couples, Opladen

Spitzley, H. (2005): „Kurze Vollzeit" - eine Grundlage für gute Arbeit, in: Zeitschrift für Gesundheitsschutz und Arbeitsgestaltung 5, S. 21-23 Wotschack, P. (2002): Zeitwohlstand als Problem sozialer Ungleichheit, in: Rinderspacher, J. (Hrsg.): Zeitwohlstand. Ein Konzept für einen anderen Wohlstand der Nation, Berlin, S. 143-163

Wotschack, P. (2007): Lebenslaufpolitik in den Niederlanden. Gesetzliche Regelungen zum Ansparen längerer Freistellungen, in: Hildebrandt, E. (Hrsg.): Lebenslaufpolitik im Betrieb. Optionen zur Gestaltung der Lebensarbeitszeit durch Langzeitkonten, Berlin, S. 241-258 